\title{
Lithium-ion battery cell-level control using constrained model predictive control and equivalent circuit models
}

\author{
Marcelo A. Xavier ${ }^{\mathrm{a}}$, M. Scott Trimboli ${ }^{\mathrm{a}, 1, *}$ \\ ${ }^{a}$ Department of Electrical and Computer Engineering, University of Colorado Colorado Springs, Colorado Springs, CO 80918, United States
}

\begin{abstract}
This paper introduces a novel application of model predictive control (MPC) to cell-level charging of a lithium-ion battery utilizing an equivalent circuit model of battery dynamics. The approach employs a modified form of the MPC algorithm that caters for direct feed-though signals in order to model near-instantaneous battery ohmic resistance. The implementation utilizes a $2^{\text {nd }}$-order equivalent circuit discrete-time state-space model based on actual cell parameters; the control methodology is used to compute a fast charging profile that respects input, output, and state constraints. Results show that MPC is well-suited to the dynamics of the battery control problem and further suggest significant performance improvements might be achieved by extending the result to electrochemical models.
\end{abstract}

Keywords: Model predictive control, lithium ion battery, equivalent circuit model, battery management systems

\section{Introduction}

A major obstacle to widespread use of electric vehicles remains the high cost and limited range associated with onboard energy storage systems (ESS). Market acceptance is further hindered by a lack of the high performance energy management systems (EMS) needed to supply the high power and energy demands presented by drivetrain electrification [1-3]. Consequently, most commercial offerings are based on hybrid energy storage systems (HESS), which aim to exploit the advantages of different ESS solutions while minimizing their respective drawbacks. All-electric vehicles (EVs) must overcome current range and cost limitations in order to gain appreciable market acceptance.

Electric vehicle energy storage is accomplished by devices that accumulate energy electrochemically to perform useful work at a later time. Examples include batteries, fuel cells, capacitors, and super (ultra) capacitors. The present work will address electrochemical storage using lithium ion batteries.

The term battery is often used to describe both a single battery cell and a multi-cell battery pack. Battery cells are defined as the smallest individual electrochemical unit, and deliver a voltage dependent on a specific cell chemistry. Battery packs are assembled from groups of cells organized into modules in series and parallel configurations. A battery pack's operation and safety is managed by a battery management system (BMS) consisting of sensor and

${ }^{*}$ Corresponding author

Email addresses: mxavier@uccs .edu (Marcelo A. Xavier), mtrimbol@uccs . edu (M. Scott Trimboli)

${ }^{1}$ Tel: +1 719255 3468; fax: +1 7192553589 
control circuitry.

It is well known that lithium ion battery performance (and indeed safe operation) can be significantly affected by the choice of charging strategy employed to replenish the battery. The ability to bring a battery to a specified stateof-charge in the shortest time possible is intrinsically limited by internal electrochemical processes. Additionally, exceeding certain current rates and cell voltages can cause irreversible damage and capacity loss that will ultimately degrade long-term performance. The most widely used charging profile for lithium-ion batteries is constant-current constant-voltage (CCCV). In this scheme, the battery is first charged at a constant current level until a specified voltage limit is reached, after which the voltage limit is held constant while the current is proportionately reduced. Although $\mathrm{CCCV}$ is relatively easy to apply, it is often based on conservative voltage limits and thus may not take full advantage of the true operating range of the battery.

Alternative charging strategies have begun to appear in the literature with some of the most promising motivated by control theory. Recent work by Hu et al. [4] devises a multi-objective optimization problem to trade-off minimization of charge time with energy loss by utilizing a linear quadratic regulator (LQR) problem formulation and a simple equivalent circuit model. Bashash, et al. [5] utilize multiple objectives to obtain vehicle charge pattern optimization between energy cost and battery longevity, while other approaches have included fuzzy logic [6] and consecutive orthogonal arrays [7], to name a few.

Most battery management control strategies address system (pack)-level control in order to achieve system level performance objectives (e.g., cell balancing). Surprisingly little has been done at the cell-level to improve overall battery performance and extend lifetime. One approach seemingly well-suited to this task is model predictive control (MPC), which is gaining popularity throughout industry [8]. In [9] the authors propose a nonlinear model predictive control approach to minimize the charging time of a lithium ion battery based on a complex underlying electrochemical model. Employing a 'look-ahead' strategy, MPC can foresee dynamic changes before they happen and efficiently compute stepwise-optimal input control to achieve a quadratic performance objective. More importantly however, MPC is able to conform to hard constraints imposed on designated problem variables. This feature makes MPC particularly appealing for the battery control problem, where respecting certain voltage and current limits can be shown to influence both instantaneous and long-term cell performance [10].

MPC has shown promise in application to hybrid system performance; for example, [11] and [12] demonstrate a fuel cell - UC configuration, and [13] and [14] use a fuel cell - battery set. These works exploit a degree of freedom introduced by a secondary energy source (either battery or ultracapacitor) in order to optimize operation of hydrogen fuel cells; in this case, constraints are imposed to avoid a detrimental oxygen starvation condition. A power flow control solution is presented in [13] and [14], while control on the current applied is presented in both [11] and [12].

The effectiveness of cell-level control is ultimately limited by the underlying mathematical model of battery cell dynamics. Existing cell-level implementations rely almost exclusively on equivalent circuit models of battery dynamics due to their inherent simplicity and general effectiveness. Related work was accomplished by Moura et al. [15] where the problem of film growth in Li-ion battery packs was addressed via switching. there, he authors propose 
an unequal charging profile obtained through switches controlled by deterministic dynamic programming (DDP) and DDP-inspired algorithms. Although showing effective reduction in film growth, the approach is computationally demanding.

Previous work by Plett ([16],[17]) has shown successful implementation of an Extended Kalman Filter (EKF) to estimate cell SOC using equivalent circuit models. For applications where it is of interest to limit SOC during operation, it is suggested that MPC may be used to optimize selected measures of cell performance while respecting such limits.

To that end, it is of interest to examine what can be achieved applying MPC techniques to the control of a battery cell using an equivalent circuit model. Specifically, this paper examines the potential efficacy of MPC to carefully regulate input current when applied to the problem of "fast charge" of a battery cell. In order to clearly illustrate the behavior of MPC with fundamental battery cell-level dynamics, we confine our treatment to discrete-time equivalent circuit cell models representing linear, time-invariant systems. Extensions to reduced-order physics-based models will follow in a subsequent paper.

This paper is organized as follows. In Section 2 we review the basics of battery cell modeling and develop the expressions defining the equivalent circuit model forming the basis of this study. Section 3 introduces the principles of Model Predictive Control and details the modifications employed to incorporate a direct feed-through term. Section 4 formulates the fast-charge optimization problem and Section 5 presents results.

\section{Battery Cell Modeling Formulation}

\subsection{Electrochemistry basics}

Vehicle autonomy and reliable performance depend heavily on accurate knowledge of a battery's internal state. When used together with 'smart' controls, dynamic state information can enable significant improvements in battery life and driving range. However, understanding the fundamental processes driving cell behavior is a critical first step to realizing these gains.

Candidate battery technologies for drivetrain electrification include: lead acid, nickel metal hydride (NiMH), and lithium ion (Li-ion). Li-ion batteries have emerged as the candidate of choice due to their high specific energy, low self-discharge rates and long cycle life. Additionally, lithium-ion cells show no memory effect and the high open open circuit voltage characteristic allows them to provide the same power at lower current, or by using a reduced number of cells compared to $\mathrm{NiMH}$ and $\mathrm{NiCd}$.

\subsection{Lithium-ion battery operation}

A battery produces electricity by releasing stored potential energy through an electrochemical process. Typical cells consist of three basic elements: a positive electrode, a negative electrode and and electrolyte material. The two electrodes are usually made of different substances, both of which chemically react with the electrolyte as ionic 
bonds $^{2}$. A separator material electrically isolates the positive and negative electrodes to avoid self discharge of the cell. Lithium-ion batteries work differently from other electrochemical cells in that they depend on an "intercalation" mechanism rather than a standard redox reaction. Intercalation involves the insertion of lithium ions into the crystalline lattice of the host electrode without altering its crystal structure.

Presently, most commercial Li-ion cells use some form of graphite $\left(\mathrm{C}_{6}\right)$ for the negative electrode material; a commonly used material for the positive electrodes is $\mathrm{Li}_{\mathrm{x}} \mathrm{CoO}$. However, since this material encounters difficulties in scaling, viable candidate cathode materials also include $\mathrm{Li}_{\mathrm{X}} \mathrm{Mn}_{2} \mathrm{O}_{4}$ and $\mathrm{Li}_{\mathrm{X}} \mathrm{FePO}_{4}$. Each electrode is in electrical contact with a current collector, which transports the electrons from the solid electrode material to the external circuit. Typically, the negative current collector is made of copper and the positive current collector is comprised of aluminum. The electrolyte in a Li-ion cell can be a solid ionically conductive polymer or a conductive liquid. The electrolyte conducts lithium ions between the electrodes when a battery conducts an electric current through an external circuit. Lithium is stored in the electrodes as a neutrally charged atomic form while $\mathrm{Li}_{+}$ions move through the electrolyte. Lithium enters the surface of an electrode particle, but diffuses inward to equalize the concentration of lithium within the electrode (see Fig. 1).

[Figure 1 about here.]

During cell discharge, the electrochemical potential energy at the negative electrode favors a chemical process that releases electrons into the external circuit and positively charged ions into the electrolyte, thus lithium ions are dissociated from the negative electrode and migrate across the electrolyte to be inserted into the crystal structure of the positive electrode. Simultaneously, due to the electrochemical potential at the positive electrode, compensating electrons traverse the external circuit and are accepted by the positive electrode to balance the reaction. This chemical process also allows the positive electrode to accept positively charged ions from the electrolyte. The process is completely reversible. Thus the lithium ions pass back and forth between the electrodes during charging and discharging.

\subsection{Equivalent circuit formulation}

The electrochemical dynamic relationship for a Li-ion cell is fundamentally a function of current, voltage, and temperature. Mathematical models of battery dynamics used in most model-based estimators and model-based controllers fall into two broad categories: (1) empirical models, which include black box [18] and equivalent circuit models (ECMs) [19]; and (2) first principles, physics-based models [20, 21].

The equivalent circuit model is a highly simplified representation based only loosely on electrochemistry [19]. The model is described by a circuit of resistors and capacitors, the values for which are typically found via empirical system-identification experiments. The ECM is considerably simpler than a first principles model, and generally amenable to efficient computation compared to full-order physics-based models. Nonetheless, ECM's have important

\footnotetext{
${ }^{2} \mathrm{An}$ ionic bond is a type of chemical bond formed due to the attraction between an atom that has lost one or more electron and an atom that has gained one or more electrons.
} 
limitations. Since the internal model variables are currents and voltages describing circuit behavior, dynamic electrochemical properties of the cell cannot be gleaned from an ECM. Regardless, the ECM is widely used in industry and for that reason will serve as an important first step in the characterization of cell-level performance using model predictive control techniques.

A number of different topologies are often used for modeling battery cells with equivalent circuits. A comprehensive study is presented in [22] in which a comparison of twelve equivalent circuit models for Li-ion batteries is performed. Among those examined, numerical results point to a superior performance of the first-order RC for LiNMC cells, while the first-order RC model with one-state hysteresis appears to be the best choice for LiFePO4 cells. Due to its overall simplicity, the first-order RC, also known in the literature as the Thevenin model, was selected for this work. The Thevenin model consists of a single resistance and one RC ladder element (see Fig. 2). Resistance $R_{0}$ represents the ohmic resistance generated from the instantaneous response obtained when a load is connected to the circuit. The single RC ladder models a diffusion process, where $R$ is the polarization resistance, and $C$ is the electric double layer capacitor, which is used to describe the transient portion of the response realized during charging and discharging.

The open circuit voltage $(\mathrm{OCV})$ is represented by a state-of-charge dependent ideal voltage source, OCV $(z(t))$. The SOC/OCV relation is typically nonlinear and obtained from a look-up table.

[Figure 2 about here.]

Utilizing a discrete-time representation, and assuming that the input current $i(t)$ is held constant over each sampling interval, the state-space system can be described by the following vector-matrix expression:

$$
\begin{aligned}
{\left[\begin{array}{c}
z_{k+1} \\
v_{C_{1}, k+1}
\end{array}\right] } & =\left[\begin{array}{cc}
1 & 0 \\
0 & \exp \left(-\frac{\Delta T}{R_{1} C_{1}}\right)
\end{array}\right]\left[\begin{array}{c}
z_{k} \\
v_{C_{1}, k}
\end{array}\right]+\left[\begin{array}{c}
-\frac{\eta_{k} \Delta T}{Q} \\
R_{1}\left(1-\exp \left(-\frac{\Delta T}{R_{1} C_{1}}\right)\right)
\end{array}\right] i_{k} \\
v_{k} & =\left[\begin{array}{ll}
0 & -1
\end{array}\right]\left[\begin{array}{c}
z_{k} \\
v_{C_{1}, k}
\end{array}\right]+\left[-R_{0}\right] i_{k}+\operatorname{OCV}\left(z_{k}\right) .
\end{aligned}
$$

A temperature dependance may also be added to the open-circuit voltage function since the cell's OCV is a static function of temperature in addition to $\mathrm{OCV}$, i.e., $\mathrm{OCV}\left(z_{k}, T_{k}\right)$. It is important to note that the presence of the ohmic resistance contributes a direct feed-through term $\left[-R_{0}\right]$ to the state-space output equation. This will necessitate a slight modification to the standard model predictive control formulation.

\section{Model Predictive Control}

\subsection{Fundamental principles}

Model predictive control belongs to a class of computer control algorithms that make use of an explicit process model to predict the future response of a plant. Generally speaking, at each sampling interval MPC attempts to optimize future plant behavior by computing a sequence of future manipulated variables where only the first input in the optimal sequence is used. The entire calculation is then repeated at subsequent intervals. MPC was originally 
developed to meet the specialized control needs of power plants and petroleum refineries as an alternative algorithm to the conventional proportional integral derivative (PID) control approach and remains as the only advanced control technique that has had widespread impact on industrial process control [23]. This is largely due to the fact that it is the only control methodology that can deal explicitly with real-world constraints since operation near constraint boundaries is often necessary for the most profitable and efficient system operation.

\subsection{Standard MPC state-space model}

The following development assumes a linearized, discrete-time, state-space model of the plant. For simplicity, we will initially assume there is no direct feed-through term, i.e., $D=0$ :

$$
\begin{aligned}
\mathbf{x}_{m, k+1} & =A_{m} \mathbf{x}_{m, k}+B_{m} \mathbf{u}_{k} \\
\mathbf{y}_{k} & =C_{m} \mathbf{x}_{m, k},
\end{aligned}
$$

where $\mathbf{x}$ is an $n$-dimensional state vector, $\mathbf{u}$ is an $l$-dimensional input vector, and $\mathbf{y}$ is an $m_{y}$-dimensional vector of measured outputs. The sub-index $k$ is the time sampling instant, and the notation with the subscript $m$ is used as a reference to the "original" system model and differentiates it from the augmented model that will be presented next.

Most implementations introduce integral action implicitly in order to eliminate steady-state error by modifying the state-space model through a differencing operation performed on both sides of Eq (3). This gives:

$$
\Delta \mathbf{x}_{m, k+1}=A_{m} \Delta \mathbf{x}_{m, k}+B_{m} \Delta \mathbf{u}_{k}
$$

where we denote the first difference of the state variable by $\Delta \mathbf{x}_{m, k+1}=\mathbf{x}_{m, k+1}-\mathbf{x}_{m, k}$ and $\Delta \mathbf{x}_{m, k}=\mathbf{x}_{m, k}-\mathbf{x}_{m, k-1}$ and similarly for the control variable by $\Delta \mathbf{u}_{k}=\mathbf{u}_{k}-\mathbf{u}_{k-1}$.

The corresponding expression for the output $\mathbf{y}_{k}$ is then computed by first defining a new augmented state vector:

$$
\mathbf{x}_{k}=\left[\frac{\Delta \mathbf{x}_{m, k}}{\mathbf{y}_{k}}\right] \text {. }
$$

and noting that

$$
\begin{aligned}
\mathbf{y}_{k+1}-\mathbf{y}_{k} & =C_{m}\left(\mathbf{x}_{m, k+1}-\mathbf{x}_{m, k}\right)=C_{m} \Delta \mathbf{x}_{m, k+1} \\
& =C_{m} A_{m} \Delta \mathbf{x}_{m, k}+C_{m} B_{m} \Delta \mathbf{u}_{k} .
\end{aligned}
$$

Combining Eqs. (5) and (6) the augmented state-space model can be written as:

$$
\begin{aligned}
\underbrace{\left[\frac{\Delta \mathbf{x}_{m, k+1}}{\mathbf{y}_{k+1}}\right]}_{\mathbf{x}_{k+1}}= & \underbrace{\left[\begin{array}{c|c}
A_{m} & 0_{m}^{T} \\
C_{m} A_{m} & I_{q \times q}
\end{array}\right]}_{A} \underbrace{\left[\frac{\Delta \mathbf{x}_{m, k}}{\mathbf{y}_{k}}\right]}_{\mathbf{x}_{k}}+\underbrace{\left[\frac{B_{m}}{C_{m} B_{m}}\right]}_{B} \Delta \mathbf{u}_{k} \\
\mathbf{y}_{k} & =\underbrace{\left[0_{m} \mid I_{q \times q}\right.}_{C}] \underbrace{\left[\frac{\Delta \mathbf{x}_{m, k}}{\mathbf{y}_{k}}\right]}_{\mathbf{x}_{k}},
\end{aligned}
$$

where $q$ denotes the number of outputs. 


\subsection{Discrete-time state-space MPC incorporating feedthrough}

Standard forms of the MPC algorithm (e.g., [24]) assume a strictly causal system, which implies $D=0$ in the statespace description. However, as previously stated, mathematical models of battery dynamics yield an output equation that is a function of both current states and present input; the latter introduces the direct feed-through component. Since the instantaneous ohmic resistance exhibited by battery cells can be significant, it is important to take this aspect into explicit account when configuring the cell model for controller design. Consequently, we shall introduce a modification to the standard algorithm in order to cater for the ohmic resistance effect. This is accomplished by leveraging the work of Ordys and Pike [25], where we first consider the SISO system described by the following linear discrete-time, state-space equations:

$$
\begin{aligned}
\mathbf{x}_{m, k+1} & =A_{m} \mathbf{x}_{m k}+B_{m} u_{k} \\
y_{k} & =C_{m} \mathbf{x}_{m k}+D_{m} u_{k} .
\end{aligned}
$$

Re-defining the state vector from Eq. (8) as:

$$
\chi_{k}=\left[\mathbf{x}_{m k} \mid u_{k}\right]^{T} .
$$

the system in Eq. (8) can be re-written in augmented form as

$$
\begin{aligned}
\chi_{k+1} & =\tilde{A} \chi_{k}+\tilde{B} \Delta u_{k+1} \\
y_{k} & =\tilde{C} \chi_{k},
\end{aligned}
$$

where we now define

$$
\tilde{A}=\left[\begin{array}{c|c}
A_{m} & B_{m} \\
\hline 0 & I
\end{array}\right], \tilde{B}=\left[\begin{array}{c}
0 \\
\hline I
\end{array}\right], \tilde{C}=\left[C_{m} \mid D_{m}\right], \text { and } \Delta u_{k+1}=u_{k+1}-u_{k} .
$$

This form allows the standard implementation machinery to be used on the re-defined quantities.

\subsection{Predictive Control Algorithm}

Unlike standard optimal control formulations which compute control magnitudes, MPC control inputs are defined in terms of their step-wise increments:

$$
\Delta u_{k}, \Delta u_{k+1}, \ldots, \Delta u_{k+N_{c}}
$$

where $k$ represents a particular time step and $N_{c}$ is a user-defined control horizon. This construction assumes that all future control input increments beyond the control horizon up to a prediction horizon $N_{p}$ (where $N_{p} \geq N_{c}$ ) are equal to zero, which implies that control inputs from $N_{c}$ to $N_{p}$ are held constant (i.e., are held equal to $u_{k_{i}+N_{c}}$ ). Of course the information contained in augmented state vector $\chi_{k+i}$ allows predictions beyond $N_{p}$, but for computational effeciency it is customary to limit the prediction horizon as performance will allow. 
Future state variable vectors are stacked into the matrix

$$
\chi=\left[\begin{array}{llllll}
\chi_{k+1 \mid k} & \chi_{k+2 \mid k} & \cdots & \chi_{k+m \mid k} & \cdots & \chi_{k+N_{p} \mid k}
\end{array}\right]^{T},
$$

where $\chi_{k+m \mid k}$ is the predicted state vector at time $k+m$ with given current plant information at time increment $k$.

Based on the state-space model given in Eq. (7) and on the future state variables defined in Eq. (10), the predicted output variables are propagated in time and assembled into a compact matrix as

$$
\mathbf{Y}_{k, N_{p}}=\Phi \tilde{A} \chi_{k}+G \Delta \mathbf{U}_{k, N_{c}}
$$

where, $\mathbf{Y}_{k, N_{p}}=\left[\begin{array}{lllll}\mathbf{y}_{k+1 \mid k} & \mathbf{y}_{k+2 \mid k} & \mathbf{y}_{k+3 \mid k} & \ldots & \mathbf{y}_{k+N_{p} \mid k}\end{array}\right]^{T}$ is the sequence of future predicted outputs, $\Delta \mathbf{U}_{k, N_{c}}=$ $\left[\begin{array}{lllll}\Delta u_{k} & \Delta u_{k+1} & \Delta u_{k+2} & \ldots & \Delta u_{k+N_{c}}\end{array}\right]^{T}$ is the sequence of future control inputs, and data matrices $\Phi$ and $G$ are defined as

$$
\Phi=\left[\begin{array}{c}
\tilde{C} \\
\tilde{C} \tilde{A} \\
\tilde{C} \tilde{A}^{2} \\
\vdots \\
\tilde{C} \tilde{A}^{N_{p}-1}
\end{array}\right], G=\left[\begin{array}{ccccc}
\tilde{C} \tilde{B} & 0 & 0 & \cdots & 0 \\
\tilde{C} \tilde{A} \tilde{B} & \tilde{C} \tilde{B} & 0 & \cdots & 0 \\
& \tilde{C} \tilde{A} \tilde{B} & \tilde{C} \tilde{B} & \cdots & 0 \\
\vdots & & & & \\
\tilde{C} \tilde{A}^{N_{p}-1} \tilde{B} & \tilde{C} \tilde{A}^{N_{p}-2} \tilde{B} & \tilde{C} \tilde{A}^{N_{p}-3} \tilde{B} & \cdots & \tilde{C} \tilde{A}^{N_{p}-N_{c}} \tilde{B}
\end{array}\right]
$$

\subsection{Quadratic optimization problem}

For a given set-point $r_{k}$ at sample time $k$, the control objective is to find the best input sequence $\Delta \mathbf{U}$ such that a specified cost function is minimized. First, let

$$
\mathbf{R}_{s}=\bar{R}_{s} r_{k}
$$

be the vector that contains the step-wise set-point information, where $\bar{R}_{s}=\left[\begin{array}{lllll}1 & 1 & 1 & \cdots & 1\end{array}\right]^{T}$ is a vector of length $N_{p}$. Next, define cost function $J$ as

$$
J_{k}=\left(\mathbf{Y}_{k}-\mathbf{R}_{s}\right)^{T} Q\left(\mathbf{Y}_{k}-\mathbf{R}_{s}\right)+\Delta \mathbf{U}_{k, N_{c}}^{T} \bar{R} \Delta \mathbf{U}_{k, N_{c}},
$$

where the first term aims to minimize errors between the predicted output and the set-point signal while the second term reflects weighting placed on the size of $\Delta \mathbf{U}$. Q is a positive semi-definite matrix. The weighting matrix $\bar{R}=$ $r_{w} I_{N_{c} \times N_{c}}\left(r_{w} \geq 0\right)$, where $r_{w}$ may be used as a tuning parameter to affect the desired closed-loop performance.

The optimal $\Delta \mathbf{U}$ that minimizes the cost in Eq. (12) is found as the vector solution of the stationarity condition

$$
\frac{\partial J_{k}}{\partial \Delta \mathbf{U}_{k}}=0
$$

and is computed as

$$
\Delta \mathbf{U}_{k, N_{c}}^{*}=\left(G^{T} G+\lambda I\right)^{-1} G^{T}\left(\mathbf{R}_{s}-\Phi \tilde{A} \chi_{k}\right) .
$$

Optimal parameter vector $\Delta \mathbf{U}_{k, N_{c}}^{*}$ contains future control values computed for time indices $k$ to $k+N_{c}$; though the receding horizon control principle stipulates only the first sample of the sequence is adopted as the current control input. The procedure is repeated at the next time sample. As mentioned previously, this formulation assumes all control increments beyond the control horizon (up to the prediction horizon) are set equal to zero. 


\section{Applying MPC to improve performance of a Li-ion battery cell}

\subsection{Fast-Charge Problem}

MPC will first be applied to the battery fast-charge problem incorporating hard system constraints. The traditional fast-charge problem consists of finding the input current time profile required to bring a battery cell from an initial state-of-charge to a specified final value in the shortest possible time, i.e.,

$$
\min _{I_{a p p}}[\text { time to charge }] .
$$

This problem is of the min-time optimal control type, and when real-world constraints are imposed on input current and cell terminal voltage, it produces the well-known CCCV profile. Min-time problems are notoriously difficult to solve in practice; so for MPC implementation, we instead fashion a cost function designed to bring about essentially the same result through a pseudo min-time problem:

$$
J_{k}=\sum_{j=0}^{N_{p}}\left\{\left(z_{k+j+1}-r_{k+j+1}\right)^{T}\left(z_{k+j+1}-r_{k+j+1}\right)+\lambda\left(\Delta u_{k+j+1}\right)^{T}\left(\Delta u_{k+j+1}\right)\right\} .
$$

Here we set $Q=I$, and $\bar{R}=\lambda$ (scalar-valued for single input systems). In essence, the cost function is designed to minimize the normed distance between the measured SOC and the output reference value while penalizing the magnitude of the input current rate. The investigation involves de-tuning a (non-zero) penalty on the current rate in such a way that it reaches a near-optimal solution as we allow the current to achieve large step-wise increments.

\subsection{Equivalent circuit cell model}

\subsubsection{Cell specifications}

The present study utilizes a model of a rechargeable lithium-ion polymer battery manufactured by LG Chem, Ltd. [26] which has a nominal capacity of $6000 \mathrm{mAh}$ and a nominal voltage of $3.85 \mathrm{~V}$. The model parameters were obtained through a data-fitting process applied to input-output data obtained from controlled battery cell tests [27]. The identification results for a Thevenin equivalent circuit model assuming a cell efficiency of $\eta=0.997$ and operating temperature of $25^{\circ} \mathrm{C}$ are shown in Table 1 .

\subsubsection{State-space model}

Introducing the cell identification results of Table 1 into Eqs. (2.10) and (2.11), gives a second-order, discrete-time state-space representation of the equivalent circuit model:

$$
\begin{aligned}
{\left[\begin{array}{c}
z_{k+1} \\
v_{C_{1}, k+1}
\end{array}\right] } & =\left[\begin{array}{cc}
1 & 0 \\
0 & 0.9940
\end{array}\right]\left[\begin{array}{c}
z_{k} \\
v_{C_{1}, k}
\end{array}\right]+\left[\begin{array}{c}
-0.5413 \times 10^{-4} \\
0.9279 \times 10^{-4}
\end{array}\right] i_{k} \\
v_{k} & =\left[\begin{array}{ll}
0 & -1
\end{array}\right]\left[\begin{array}{c}
z_{k} \\
v_{C_{1}, k}
\end{array}\right]+[-0.0121] i_{k}+\operatorname{OCV}\left(z_{k}\right) .
\end{aligned}
$$


The OCV - SOC relationship is inferred via a lookup table. Since SOC is designated the output to be controlled, we write an output equation of the form

$$
z_{k}=\left[\begin{array}{ll}
1 & 0
\end{array}\right]\left[\begin{array}{c}
z_{k} \\
v_{C_{1}, k}
\end{array}\right] .
$$

[Table 1 about here.]

\subsection{Control Design}

Following the development of section 3.2 we first augment the state-space system to introduce integral action:

$$
\begin{aligned}
& \chi_{k+1}=\left[\begin{array}{cc|c}
1 & 0 & -0.5413 \times 10^{-4} \\
0 & 0.9940 & 0.9279 \times 10^{-4} \\
\hline 0 & 0 & 1
\end{array}\right] \chi_{k}+\left[\begin{array}{c}
0 \\
0 \\
\hline 1
\end{array}\right] \Delta i_{k+1} \\
& v_{k}=\left[\begin{array}{ll}
0 & -1
\end{array}-0.0121\right] \chi_{k} \\
& z_{k}=\left[\begin{array}{ll|l}
1 & 0 & 0
\end{array}\right] \chi_{k} \text {. }
\end{aligned}
$$

The principle control objective is to find the applied current profile $\left\{i_{k}\right\}$ that drives the SOC from an initial value to a desired reference while keeping terminal voltage within cell-specific operational bounds. To respect physical limits and avoid overcharge we also bound SOC at the reference target value:

$$
z_{k} \leq 0.9
$$

Moreover, the applied current is bounded at maximum values of $1 \mathrm{C}$ and $2 \mathrm{C}$ rates determined from the cell nominal capacity at $25^{\circ} \mathrm{C}$. Voltage and current constraints are summarized below:

$$
\begin{aligned}
3 \mathrm{~V} & \leq v_{k} \leq 4.2 \mathrm{~V} \\
-5.1160 \mathrm{~A} & \leq i_{k} \leq 5.1160 \mathrm{~A}, \text { for } 1 \mathrm{C} \\
-10.2320 \mathrm{~A} & \leq i_{k} \leq 10.2320 \mathrm{~A}, \text { for } 2 \mathrm{C} .
\end{aligned}
$$

Step-wise control inputs $i_{k}$ are are calculated at each time instant. For control and prediction horizons of $N_{c}$ and $N_{p}$, respectively, a sequence of control increments is computed at each time instant, $k$ :

$$
\Delta \mathbf{U}_{k, N_{c}}=\left[\begin{array}{llll}
\Delta u_{k+1} & \Delta u_{k+2} & \ldots & \Delta u_{k+N_{c}}
\end{array}\right]^{T} .
$$

The optimal control sequence minimizes the finite horizon performance index in Eq. (15) and simultaneously satisfies constraints on control amplitude, terminal voltage and SOC for future $k$ values up to the prediction horizon. 


\section{Simulation Results and Discussion}

A range of test cases was run at both $1 \mathrm{C}$ and $2 \mathrm{C}$ maximum charge rates using the following parameter sets:

$$
\begin{aligned}
\text { Control weighting } & \lambda & =\left[\begin{array}{lllll}
0.1 & 0.01 & 0.001 & 0.0001
\end{array}\right] \\
\text { Prediction horizon } & N_{p} & =\left[\begin{array}{lllll}
10 & 20 & 60 & 100 & 200
\end{array}\right] ; \\
\text { Control horizon } & N_{c} & =\left[\begin{array}{llll}
1 & 2 & 3 & 6
\end{array}\right] .
\end{aligned}
$$

Tuning among these parameters yields a solution to the $1 \mathrm{C}$ rate-limited pseudo minimum - time problem at: $N_{p}=$ $10, N_{c}=1$, and $\lambda=0.0001$ (see Figure 3 ). This parameter set delivered a CCCV charging profile that drove the cell SOC from 0.5 to 0.9 in 1471 seconds.

[Figure 3 about here.] 
For a current limit of $2 \mathrm{C}$ the tuning parameters turn out to be the same as for $1 \mathrm{C}$. Due to the higher maximum current rate, however, the total time-to-charge decreased (as expected) to 1158 seconds. The results are shown in Fig. 4.

[Figure 4 about here.]

The effect of varying the penalty weight is shown in Fig. 5 for a maximum current rate of $2 \mathrm{C}$. Here we fix the prediction and control horizons at $N_{p}=10$ and $N_{c}=1$, respectively, and vary only the weight on the control increment. Relaxing the value of the penalty weight allows the control algorithm to compute larger variations of the control increment causing the charge current to "jump" to its maximum value, bringing about a quicker system SOC response. The system performance for both $\lambda=0.001$ and $\lambda=0.0001$ appear the same; however, the computed total time to charge was slightly less for the latter case. In order to explore the influence of the weighting factor for even smaller values, we performed simulations reducing $\lambda$ down to $10^{-10}$; all delivered the same performance as for $\lambda=0.0001$, which we take as the limiting case. Conversely, it can be seen that heavy weighting on the control increment produces a predictably sluggish response.

[Figure 5 about here.]

The effect of changing the control and prediction horizons for the 2C rate-limited case are shown in Figs. 6 and 7 , respectively. Clearly, the length of the control horizon has very little effect on performance for this system. It was observed, however, that for extremely small penalty weights (approaching zero), we begin to experience numerical anomalies for larger values of $N_{c}$; in particular, the algorithm fails to respect hard constraints on the control amplitude. It turns out that this behavior results from ill-conditioning of the quasi-Hessian matrix $\left(G^{T} G+\lambda I\right)^{-1}$ that appears in the optimal control sequence calculation in Eq. (13). As $\lambda$ approaches zero, the matrix $G^{T} G+\lambda I \rightarrow G^{T} G$; the rows of the latter matrix comprising shifted versions of the discrete-time impulse responses for the modeled system. As $N_{c}$ is made large, the rows become increasingly linearly dependent, the matrix becomes ill-conditioned, and hence numerically unreliable on inversion. This creates numerical issues when computing the optimal $\mathbf{\Delta} \mathbf{U}$ during the optimization steps of the Hildreth quadratic programming algorithm. Note that this problem is mitigated by selecting control horizon values $N_{c}$ that are small relative to $N_{p}$. Indeed when $N_{c}=1$, this term become scalar, and the inverse is guaranteed

Fig. 6 shows the influence of the control horizon on SOC performance for a relatively large control input weight of $\lambda=0.1$. There is no apparent difference as we vary the control horizon while keeping the prediction horizon and the penalty weight constant. However, the computed total time-to-charge yields a slight reduction from 1740 seconds for $N_{c}=1$ to 1726 seconds for $N_{c}=6$. This implies that for certain penalty weights, increasing the control horizon may improve overall performance.

[Figure 6 about here.]

Figure 7 shows that large prediction horizons slow the speed of response. This behavior is primarily due to the assumption of a constant control amplitude beyond the control horizon (up to the the prediction horizon). The predicted 
presence of this far-future control input causes the controller to generate a less aggressive input in near-future time. Further research is on-going to investigate alternative strategies for the far-future control assumption with a view to improving near-future performance while maintaining short control horizons.

[Figure 7 about here.]

\section{Conclusion}

An application of constrained MPC is presented that generates the CCCV input charge current profile for fast charging of a lithium-ion battery cell. The algorithm accommodates direct feed-through to correctly model ohmic resistance and utilizes a second-order, discrete-time equivalent circuit model for prediction. Model parameters were obtained from a data-fitting process utilizing input-output experiments. The MPC cost function is configured and tuned to deliver a solution to a "pseudo minimum-time" optimization problem under hard constraints imposed on input current, terminal voltage and maximum state-of-charge. A parametric study is presented which examines the influence of key tuning parameters on controlled system performance. Constrained MPC exhibited stable and acceptable performance for the fast-charge problem over a wide range of tuning parameters.

\section{Acknowledgement}

The information, data, or work presented herein was funded in part by the Advanced Research Projects AgencyEnergy (ARPA-E), U.S. Department of Energy, under Award Number DE-AR0000271.

Financial support for the research reported in this paper has also been received from the General Motors/University of Michigan Advanced Battery Coalition for Drivetrains (GM/UM ABCD), to which the University of Colorado Colorado Springs is a subcontractor.

This work used the EAS Data Center or EAS Cloud, which is supported by the College of Engineering and Applied Science, University of Colorado Colorado Springs.

Disclaimer: The information, data, or work presented herein was funded in part by an agency of the United States Government. Neither the United States Government nor any agency thereof, nor any of their employees, makes

any warranty, express or implied, or assumes any legal liability or responsibility for the accuracy, completeness, or usefulness of any information, apparatus, product, or process disclosed, or represented that its use would not infringe privately owned rights. Referenced herein to any specific commercial product, process, or service by trade name, trademark, manufacturer, or otherwise does not necessarily constitute or imply its endorsement, recommendation, or favoring by the United States Government or any agency thereof. The views and opinions of authors expresses herein do not necessarily state or reflect those of the United States Government or any agency thereof. 


\section{References}

\section{References}

[1] S. M. Lukic, A. Emadi, IEEE Transactions on Vehicular Technology 53 (2004) 385-389.

[2] A. Affanni, A. Bellini, G. Franceschini, P. Guglielmi, C. Tassoni, IEEE Transactions on Industrial Electronics 52 (2005) 1343-1349.

[3] C. Chan, Y. Wong, IEEE Power and Energy Magazine 2 (2004) 24-33.

[4] X. Hu, S. Li, H. Peng, F. Sun, Journal of Power Sources 239 (2013) 449-457.

[5] S. Bashash, S. J. Moura, J. C. Forman, H. K. Fathy, Journal of Power Sources 196 (2011) 541-549.

[6] J. Jiang, C. Zhang, J. Wen, W. Zhang, S. Sharkh, IEEE Transactions on Vehicular Technology 62 (2013) 30003009.

[7] Y.-H. Liu, C.-H. Hsieh, Y.-F. Luo, IEEE Transactions on Energy Conversion 26 (2011) 654-661.

[8] E. F. Camacho, C. B. Alba, Model predictive control, Springer, 2013.

[9] R. Klein, N. Chaturvedi, J. Christensen, J. Ahmed, R. Findeisen, A. Kojic, in: American Control Conference (ACC), 2011, pp. 382-387.

[10] M. M. U. Rehman, M. Evzelman, K. Hathaway, R. Zane, G. L. Plett, K. Smith, E. Wood, D. Maksimovic, in: Proceedings of the 2014 IEEE Energy Conversion Congress and Exposition (ECCE 2014), IEEE.

[11] A. Vahidi, A. Stefanopoulou, H. Peng, IEEE Transactions on Control Systems Technology 14 (2006) 1047-1057.

[12] A. Vahidi, A. Stefanopoulou, H. Peng, in: Proceedings of the American Control Conference, 2004., volume 1, IEEE, pp. 834-839.

[13] R. Meyer, R. A. DeCarlo, P. H. Meckl, C. Doktorcik, S. Pekarek, in: American Control Conference (ACC), 2011, IEEE, pp. 2725-2731.

[14] A. Arce, A. J. del Real, C. Bordons, Journal of Process Control 19 (2009) 1289-1304.

[15] S. J. Moura, J. C. Forman, J. L. Stein, H. K. Fathy, in: 2nd Annual Dynamic Systems and Control Conference, DSCC.

[16] G. L. Plett, Journal of Power Sources 161 (2006) 1356-1368.

[17] G. L. Plett, Journal of Power Sources 161 (2006) 1369-1384. 
[18] I. Snihir, W. Rey, E. Verbitskiy, A. Belfadhel-Ayeb, P. H. Notten, Journal of Power Sources 159 (2006) 14841487.

[19] M. W. Verbrugge, R. S. Conell, Journal of the Electrochemical Society 149 (2002) A45-A53.

[20] M. Doyle, T. F. Fuller, J. Newman, Journal of The Electrochemical Society 140 (1993) 1526-1533.

[21] T. F. Fuller, M. Doyle, J. Newman, Journal of The Electrochemical Society 141 (1994) 1-10.

[22] X. Hu, S. Li, H. Peng, Journal of Power Sources 198 (2012) 359-367.

[23] J. M. Maciejowski, Predictive Control: With Constraint, Pearson education, 2002.

[24] L. Wang, Model Predictive Control System Design and Implementation Using MATLAB, Springer Verlag, 2009.

[25] A. Ordys, A. Pike, in: Proceedings of the 37th IEEE Conference on Decision and Control, volume 4, IEEE, pp. $4740-4741$.

[26] LG Chem medium format rechargeable lithium ion polymer battery for e-bike and electric vehicle application, online: http://www . lgchem. com, accessed September 2014.

[27] G. L. Plett, Battery Management Systems: Volume 1, Modeling [draft manuscript, revision 20140807], Retrieved from http://mocha-java.uccs.edu/BMS1/, 2014.

\section{Nomenclature}

A state transition matrix of the state-space model

B input matrix of the state-space model

C output matrix of the state-space model

$C_{1} \quad$ electric double layer capacitance, $\mathrm{F}$

D input-output coupling matrix of the state-space model

G data matrix used to compute the sequence of predicted outputs

$i \quad$ applied cell current, A

$J \quad$ cost function

$k \quad$ time sampling instant

$N_{c} \quad$ control horizon

$N_{p} \quad$ prediction horizon

$Q \quad$ total capacity of a battery cell, $\mathrm{A} \mathrm{h}$ 
$r_{w} \quad$ tuning parameter to affect the desired closed-loop performance

$R_{0} \quad$ ohmic resistance, ohms

$R_{1} \quad$ polarization resistance, ohms

$\bar{R} \quad$ weighting matrix

$\mathbf{u} \quad$ input vector of state-space model

$\mathbf{X}$ state vector of state-space model linear output vector of state-space model

Y vector containing the sequence of predicted outputs

$z \quad$ battery cell state of charge (SOC)

\section{Greek}

$\eta \quad$ coulombic efficiency

$\lambda \quad$ control weighting

Ф data matrix used to compute the sequence of predicted outputs

$\chi \quad$ augmented state vector of state-space model

\section{Subscript/superscript}

pertaining to the "original" system model 


\section{List of Figures}

1 A solid particle of lithium illustrating the diffusion of $\mathrm{Li}_{+}$and movement due to electrochemical po-

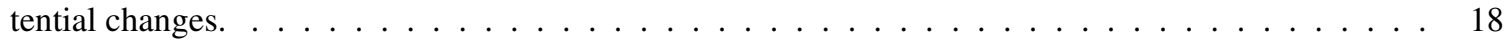

2 The Thevenin Model. . . . . . . . . . . . . . . . . . . . . . . . . . . . . . . 19

3 (a) SOC, (b) terminal voltage, and (c) charge current for $1 \mathrm{C}$ rate, $N_{p}=10, N_{c}=1$, and $\lambda=0.0001 \ldots .20$

4 (a) SOC, (b) terminal voltage, and (c) charge current for $2 \mathrm{C}$ rate, $N_{p}=10, N_{c}=1$, and $\lambda=0.0001 \ldots .21$

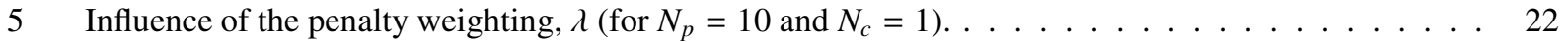

6 Influence of the control horizon, $N_{c}$ (for $N_{p}=10, \lambda=0.1$, and maximum charge current of $2 \mathrm{C}$ rate). . 23

7 Influence of the prediction horizon, $N_{p}$ (for $N_{c}=1$ and $\left.\lambda=0.0001\right) \ldots \ldots \ldots \ldots \ldots$ 


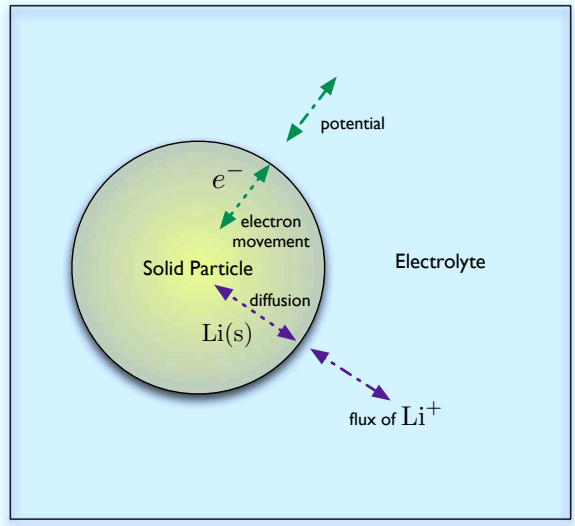

Figure 1: A solid particle of lithium illustrating the diffusion of $\mathrm{Li}_{+}$and movement due to electrochemical potential changes. 


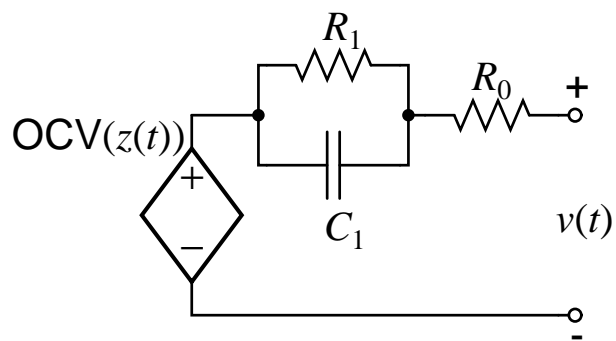

Figure 2: The Thevenin Model. 


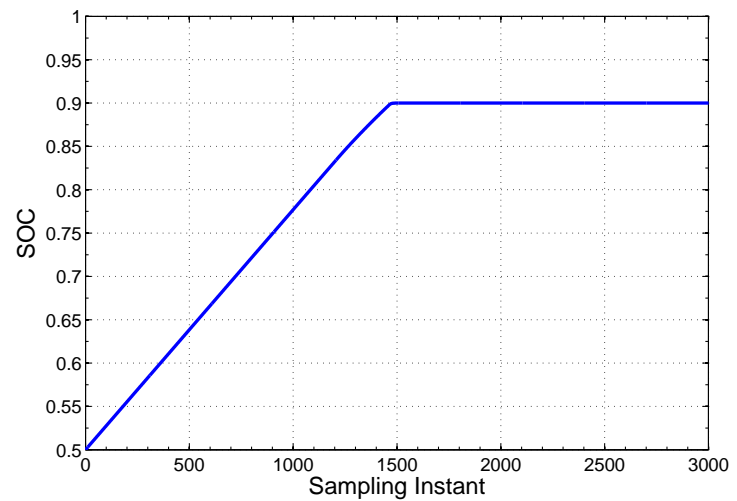

(a)

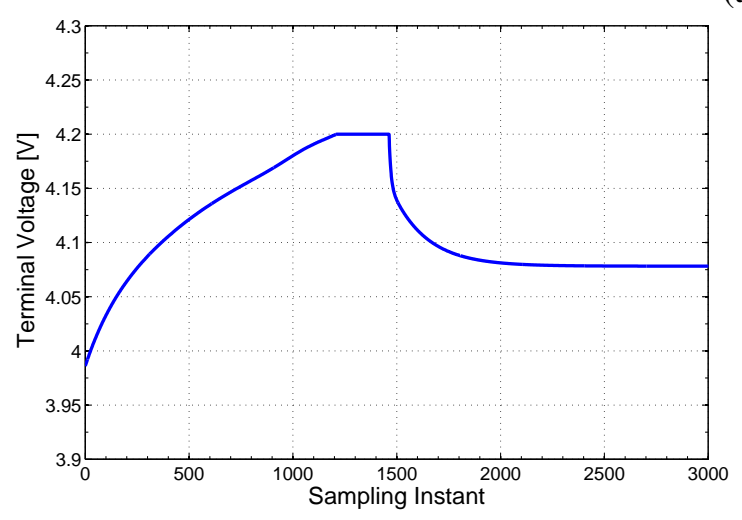

(b)

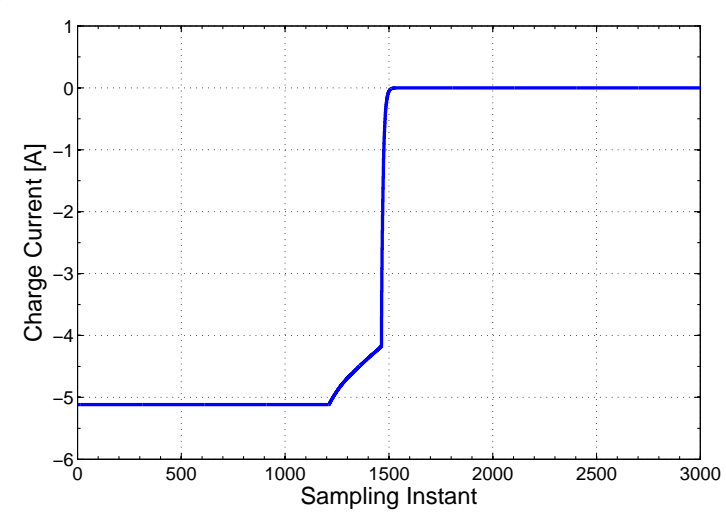

(c)

Figure 3: (a) SOC, (b) terminal voltage, and (c) charge current for 1C rate, $N_{p}=10, N_{c}=1$, and $\lambda=0.0001$. 


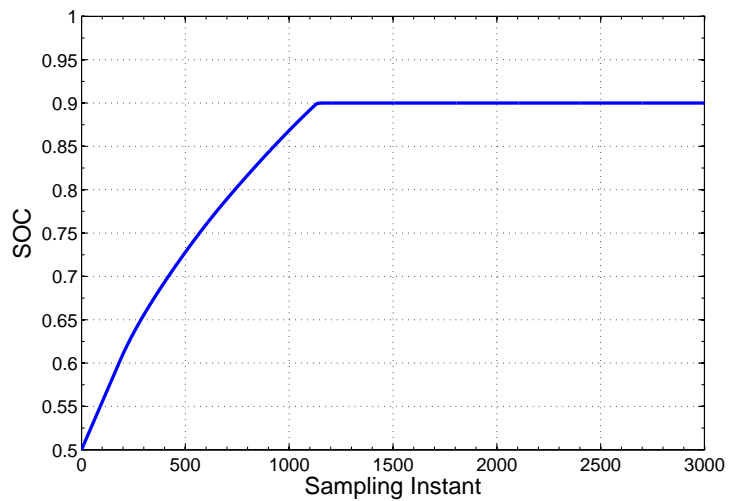

(a)

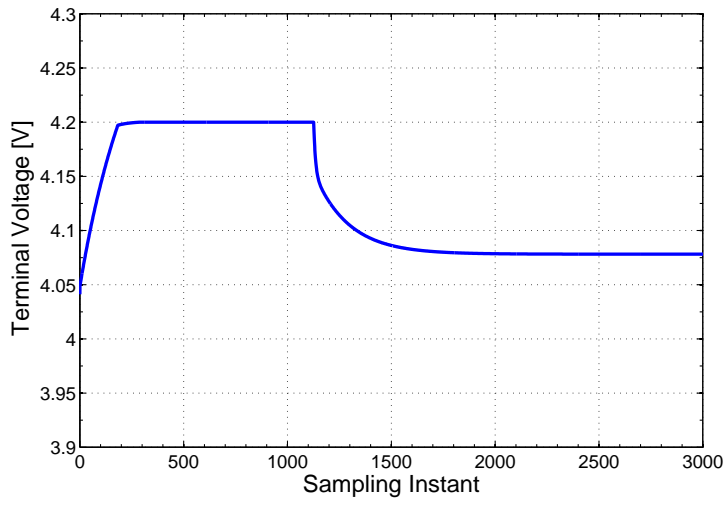

(b)

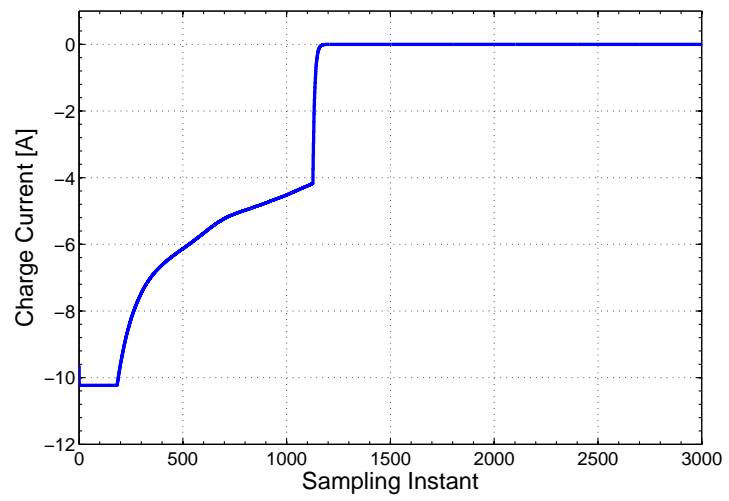

(c)

Figure 4: (a) SOC, (b) terminal voltage, and (c) charge current for $2 \mathrm{C}$ rate, $N_{p}=10, N_{c}=1$, and $\lambda=0.0001$. 


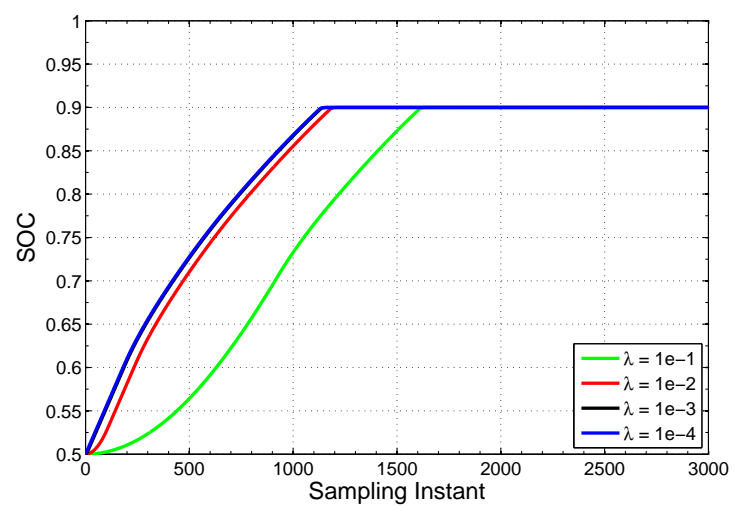

(a)

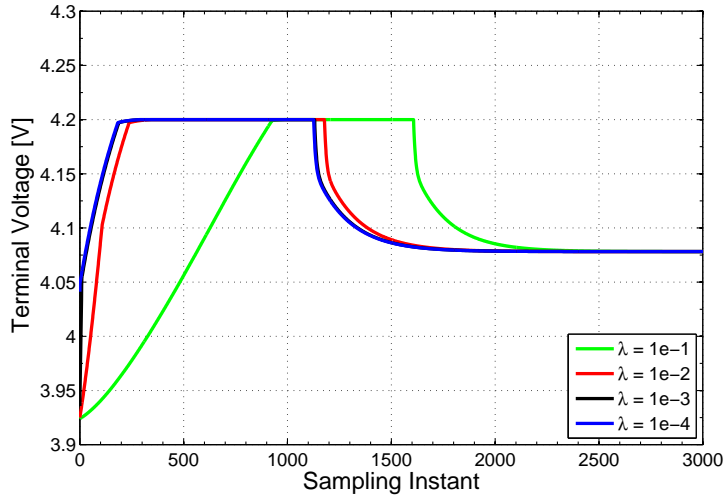

(b)

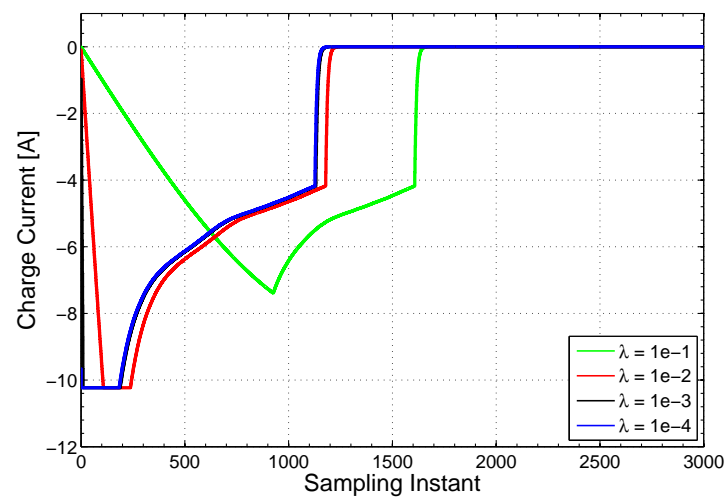

(c)

Figure 5: Influence of the penalty weighting, $\lambda$ (for $N_{p}=10$ and $N_{c}=1$ ). 


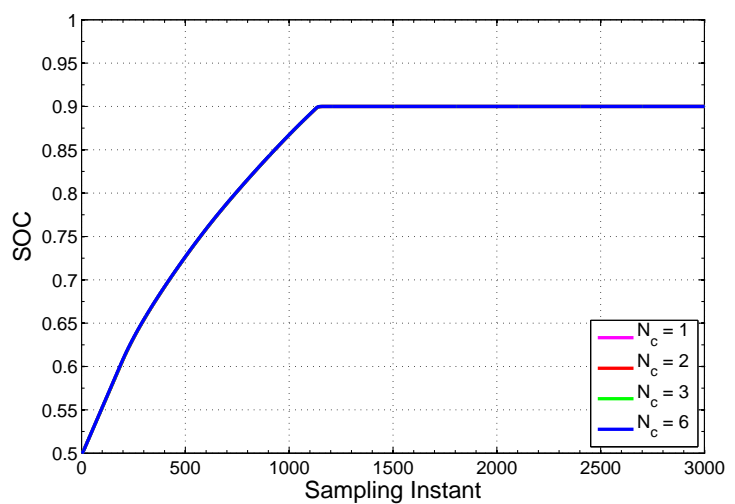

(a)

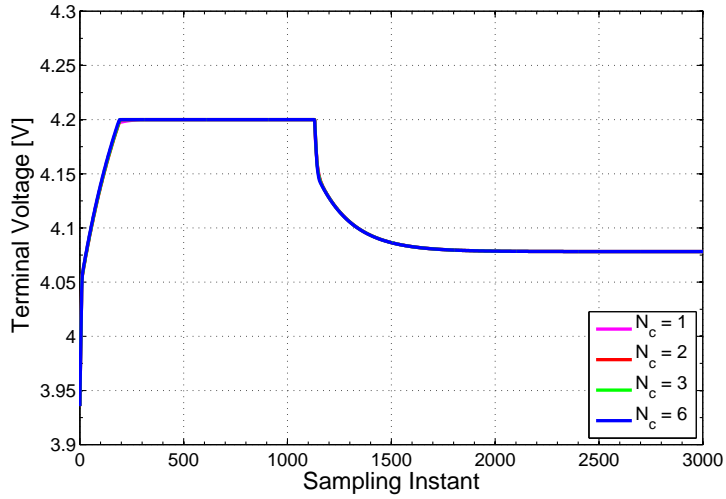

(b)

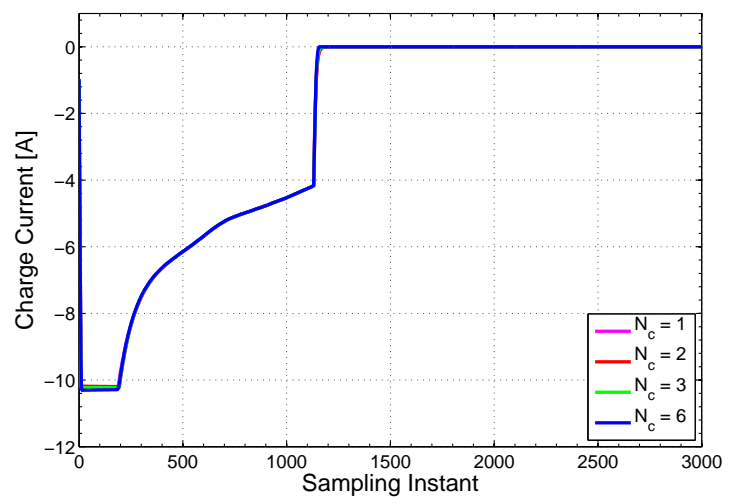

(c)

Figure 6: Influence of the control horizon, $N_{c}$ (for $N_{p}=10, \lambda=0.1$, and maximum charge current of $2 \mathrm{C}$ rate). 


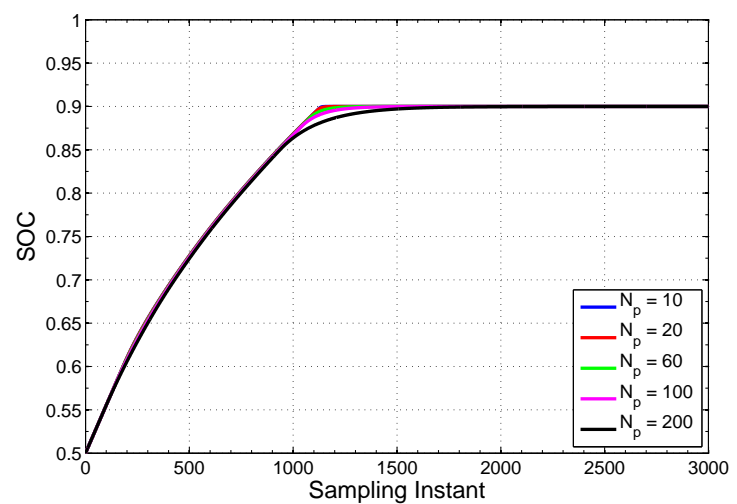

(a)

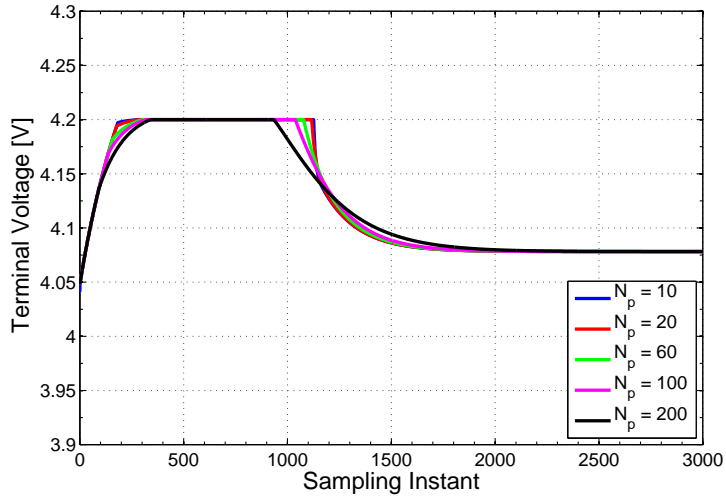

(b)

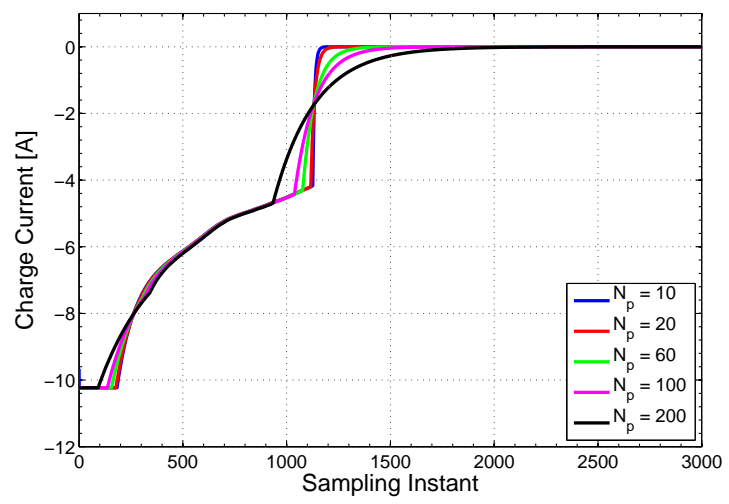

(c)

Figure 7: Influence of the prediction horizon, $N_{p}$ (for $N_{c}=1$ and $\left.\lambda=0.0001\right)$. 


\section{List of Tables}

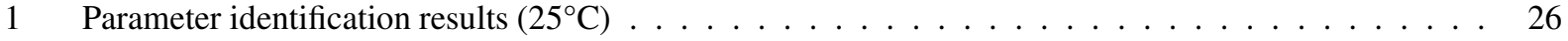


Table 1: Parameter identification results $\left(25^{\circ} \mathrm{C}\right)$

\begin{tabular}{cc}
\hline Parameter & Value \\
\hline$R_{0}$ & $0.0121 \mathrm{ohms}$ \\
$R_{1}$ & $0.0155 \mathrm{ohms}$ \\
$R_{1} C_{1}$ & $166.8685 \mathrm{sec}$ \\
$Q$ & $5.160 \mathrm{Ah}$ \\
\hline
\end{tabular}


Table 1: Parameter identification results ( $\left.25^{\prime} \mathrm{C}\right)$

\begin{tabular}{cc}
\hline Parameter & Value \\
\hline$R_{\mathrm{u}}$ & $0.0121 \mathrm{ohms}$ \\
$R_{1}$ & $0.0155 \mathrm{ohms}$ \\
$R_{1} C_{1}$ & $166.8685 \mathrm{sec}$ \\
$Q$ & $5.160 \mathrm{Ah}$ \\
\hline
\end{tabular}

